\title{
Parâmetros fisiológicos de bovinos cruzados submetidos ao estresse calórico
}

[Physiologic parameters of crossbred cattle subjected to heat stress]

\author{
F. Ferreira ${ }^{1}$, M.F.A. Pires ${ }^{2 *}$, M.L. Martinez ${ }^{2}$, S.G. Coelho ${ }^{3}$, A.U. Carvalho ${ }^{3}$, \\ P.M. Ferreira ${ }^{3}$, E.J. Facury Filho ${ }^{3}$, W.E. Campos ${ }^{3}$ \\ ${ }^{1}$ Aluna de pós-graduação - EV-UFMG \\ ${ }^{2}$ Embrapa Gado de Leite - Juiz de Fora, MG \\ ${ }^{3}$ Escola de Veterinária - UFMG \\ Caixa Postal 567 \\ 30123-970 - Belo Horizonte, MG
}

\begin{abstract}
RESUMO
Caracterizaram-se as respostas físiológicas de termorregulação em conforto e estresse calórico de bovinos cruzados $F_{2}(1 / 2$ Gir x $1 / 2$ Holandês), machos e fêmeas, com idade entre 14 e 20 meses. Os animais foram alocados, em número de cinco, e submetidos ao conforto térmico $\left(22^{\circ} \mathrm{C}\right.$ a $70 \%$ de umidade relativa) por 12 horas e ao estresse calórico $\left(42^{\circ} \mathrm{C}\right.$ e $60 \%$ de umidade relativa) durante seis horas, logo após o período de conforto, nas estações de verão e inverno, em câmara bioclimática. Nas duas etapas, foram registradas a temperatura retal (TR), a freqüência respiratória (FR) e a temperatura de superfície corporal (TSC) às 6 e 16 horas. Os animais apresentaram aumento da TR, da FR e da TSC no período de estresse calórico $(\mathrm{P}<0,05)$. $\mathrm{O}$ aumento da TR e FR foi mais acentuado no verão $(\mathrm{P}<0,05)$. A TSC não apresentou diferença entre as estações $(\mathrm{P}<0,05)$.
\end{abstract}

Palavras-chave: bovino, estresse calórico, temperatura retal, freqüência respiratória, temperatura de superfície corporal

\begin{abstract}
The thermoregulation physiological responses in thermoneutrality and heat stress of crossbred calves ( $1 / 2$ Gir $x 1 / 2$ Holstein), males and females, with age between 14 and 20 months were studied. The animals were submitted to thermoneutrality $\left(22^{\circ} \mathrm{C}\right.$ and $70 \%$ of relative humidity) and to heat stress $\left(42^{\circ} \mathrm{C}\right.$ and $60 \%$ of relative humidity) in summer and winter season's in bioclimatic chamber. Rectal temperature (TR), respiratory rate (FR) and body surface temperature (BST) were registered at 6 AM and 4 PM. It was observed increase in TR, FR and BST in the heat stress period $(P<0.05)$. TR and FR increased more in the summer than in the winter $(P<0.05)$. No difference between seasons $(P>0.05)$ was observed for $B S T$.
\end{abstract}

Keywords: cattle, heat stress, rectal temperature, respiratory rate, body surface temperature

\section{INTRODUÇÃO}

O estresse caracteriza-se pela soma de mecanismos de defesa do animal a um agente estressor. Com a evolução da bovinocultura, surgiu uma série de problemas metabólicos e de manejo, destacando-se, entre eles, o estresse calórico. A susceptibilidade dos bovinos ao estresse calórico aumenta à medida que o binômio umidade relativa e temperatura

Recebido em 15 de julho de 2005

Aceito em 30 de junho de 2006

*Autor para correspondência (corresponding author)

E-mail: fatinha@cnpgl.embrapa.br 
ambiente ultrapassa a zona de conforto térmico, o que dificulta a dissipação de calor que, por sua vez, aumenta a temperatura corporal, com efeito negativo sobre o desempenho. $\mathrm{O}$ estresse calórico promove alterações na homeostase e tem sido quantificado mediante mensuração de variáveis fisiológicas tais como, temperatura retal, freqüência respiratória e concentrações hormonais. Do ponto de vista bioclimático, mesmo para animais cruzados, considerados tolerantes ao calor, podem ocorrer alterações comportamentais e fisiológicas (Nardone, 1998).

A manutenção da temperatura corporal é determinada pelo equilíbrio entre a perda e o ganho de calor. A referência fisiológica dessa variável é obtida mediante a mensuração da temperatura retal, que pode variar de $38,1^{\circ} \mathrm{C}$ a $39,1^{\circ} \mathrm{C}$ para animais das raças de corte especializadas, de $38,0^{\circ} \mathrm{C}$ a $39,3^{\circ} \mathrm{C}$, para animais leiteiros (Robinson, 1999) e $38,0^{\circ} \mathrm{C}$ a $39,0^{\circ} \mathrm{C}$, nos bovinos adultos (Dirksen et al., 1993). A referência fisiológica para essa variável está entre 38 e $39,5^{\circ} \mathrm{C}$ sob condições termoneutras (DuPreez, 2000). A temperatura retal é usada, freqüentemente, como índice de adaptação fisiológica ao ambiente quente, pois seu aumento indica que os mecanismos de liberação de calor tornaram-se insuficientes para manter a homeotermia (Mota, 1997). Baccari Jr. (1987b) afirmou que o calor necessário para manter a temperatura corporal dos animais deriva do metabolismo e da absorção da radiação solar, direta ou indireta, enquanto a temperatura corporal depende do equilíbrio entre o calor produzido e o liberado para o ambiente.

Fatores extrínsecos podem atuar na variação da temperatura retal como a hora do dia, ingestão de alimentos e de água, estado nutricional, temperatura ambiente, densidade, sombreamento, velocidade dos ventos, estação do ano, exercício e radiação solar (Baccari Jr., 1987b; Carvalho et al., 1995).

Fatores intrínsecos estão relacionados com a individualidade, como por exemplo, idade, raça, sexo e estado fisiológico (Baccari Jr., 1971; Robinson, 1999; Stöber, 1993; Carvalho et al., 1995). Outro fator intrínseco importante na avaliação da temperatura retal é a capacidade de adaptação do animal ao ambiente. Bovinos zebuínos adaptados aos trópicos são menos sujeitos aos efeitos extremos da temperatura quando comparados aos bovinos taurinos, mais adaptados aos climas temperados (Carvalho et al., 1995).

A freqüência respiratória está sujeita a variações intrínsecas e extrínsecas. As intrínsecas caracterizam-se pelas respostas aos exercícios físicos, medo, excitação, estado físiológico e produção de leite (Wolff e Monty, 1974; Stöber, 1993; Carvalho et al., 1995; Marai et al., 1999). Fatores extrínsecos são atribuídos ao ambiente, como condições climáticas, principalmente temperatura e umidade do ar, radiação solar, velocidade dos ventos, estação do ano, hora do dia, densidade e sombreamento (Ingraham et al., 1979; Igono et al., 1985; Okantah et al., 1992; Muller e Botha, 1993; Muller et al., 1994a; Pires et al., 1998b; Marai et al., 1999). A freqüência respiratória normal em bovinos adultos varia entre 24 e 36 movimentos respiratórios por minuto (mov/min) (Stöber, 1993), mas pode apresentar valores mais amplos, entre 12 e 36 mov/min (Terra, 1993). Sob estresse térmico, a freqüência respiratória começa a elevar-se antes da temperatura retal (Bianca, 1965) e, geralmente, observa-se taquipnéia em bovinos em ambientes com temperatura elevada (Stöber, 1993; Muller et al., 1994b; Pires et al., 1998a).

A temperatura de superfície corporal depende, principalmente, das condições ambientes de umidade e temperatura do ar e vento, e das condições fisiológicas, como vascularização e evaporação pelo suor. Assim, contribui para a manutenção da temperatura corporal mediante trocas de calor com o ambiente em temperaturas amenas. Os bovinos dissipam calor para o ambiente através da pele por radiação, condução e convecção, ou seja, perda de calor sensível (Cunningham, 1999). Sob condições de estresse pelo calor, as perdas sensíveis são diminuídas e a evaporação torna-se o principal processo de perda de calor. Segundo Martello (2002), a temperatura da superfície corporal de vacas da raça Holandesa, alojadas em instalações climatizadas, pode variar de $31,6^{\circ} \mathrm{C}(6 \mathrm{~h})$ a $34,7^{\circ} \mathrm{C}$ (13h), sem indicar que o animal está sofrendo estresse pelo calor.

Este trabalho teve o objetivo de estudar as respostas fisiológicas de bovinos cruzados $F_{2}(1 / 2$ Gir $x \quad 1 / 2$ Holandês) submetidos ao estresse calórico. 


\section{MATERIAL E MÉTODOS}

Foram utilizados 71 bovinos da geração $F_{2}(1 / 2$ Gir $x \quad 1 / 2$ Holandês), provenientes dos cruzamentos entre animais $F_{1}(1 / 2$ Holandês e $1 / 2$ Gir) $x F_{1}(1 / 2$ Holandês e $1 / 2$ Gir $), 39$ machos e 32 fêmeas, com idades entre 14 e 20 meses, pesos corporais entre 350 e $400 \mathrm{~kg}$. Os animais permaneceram em piquetes com Brachiária decumbens contendo cochos para sal mineral e água, fornecidos à vontade. No dia anterior à entrada na câmara bioclimática, os animais foram trazidos dos piquetes para um local mais próximo. Dentro da câmara bioclimática, não se forneceram água ou alimentos para atenuar seus efeitos nas respostas animais. Essas condições foram citadas por McDowell (1972) como adequadas, por permitir respostas mensuráveis para todos os animais. Esse manejo foi repetido com intervalo de seis meses, abrangendo as estações de verão e inverno.

A temperatura e a umidade do ar dentro da sala foram registradas colocando-se termômetro de bulbo seco, de bulbo úmido e termômetro de globo negro em três pontos distintos para caracterizar o ambiente térmico dentro da sala durante o período experimental. $\mathrm{O}$ primeiro ponto localizava-se perto da porta, o segundo próximo ao cocho e o terceiro no fundo da sala. Calculou-se o índice de temperatura e umidade (ITU) de acordo com Kelly e Bond (1971). Na tarde anterior ao dia do tratamento, os animais eram alocados na câmara bioclimática, com capacidade para cinco animais, em condições de termoneutralidade (temperatura de $22^{\circ} \mathrm{C}$ e $70 \%$ de umidade relativa), onde permaneceram por 12 horas. $\mathrm{Na}$ manhã do dia do tratamento, às $6 \mathrm{~h}$, realizou-se a primeira coleta das características fisiológicas. Após isso, os equipamentos foram ajustados, modificando-se a temperatura para $42^{\circ} \mathrm{C}$ e umidade relativa para $60 \%$. Quando os termômetros atingiam os valores prédeterminados, às $10 \mathrm{~h}$, iniciava-se a contagem do tempo para que os animais aí permanecessem por período de seis horas. Após as seis horas de permanência, procedia-se à segunda coleta.

A temperatura retal e os movimentos respiratórios foram mensurados por dois examinadores. A temperatura retal foi obtida com o auxílio de um termômetro clínico digital inserido, aproximadamente, $7,5 \mathrm{~cm}$ no reto. No período da tarde, quando a temperatura retal ultrapassava o valor de $42^{\circ} \mathrm{C}$, houve necessidade da utilização de termômetro graduado. A freqüência respiratória foi mensurada pela contagem dos movimentos do flanco, e a temperatura de superfície corporal foi tomada em quatro pontos do corpo, cabeça, cernelha, virilha e canela, utilizando-se termômetro infravermelho, obtendo-se, ao final a média dos quatro pontos por animal.

Os dados das variáveis fisiológicas foram analisados por meio do seguinte modelo estatístico: $\mathrm{Y}_{\mathrm{ijkl}}=\mu+\mathrm{E}_{\mathrm{i}}+\mathrm{S}_{\mathrm{j}}+\mathrm{P}_{\mathrm{k}}+\mathrm{e}_{\mathrm{ijkl}}$, em que: $Y_{\mathrm{ijklm}}$ é a variável dependente; $\mu$ é a média geral; $E_{\mathrm{i}}$ é o efeito fixo de estação da medida (i=verão, inverno); $\mathrm{S}_{\mathrm{j}}$ é o efeito fixo de sexo do animal ( $\mathrm{j}=$ =macho, fêmea); $\mathrm{P}_{\mathrm{k}}$ é o efeito fixo de período ( $\mathrm{k}=$ manhã, tarde) e $\mathrm{e}_{\mathrm{ijkl}}$ é $\mathrm{o}$ erro associado à cada observação com $\sim \mathrm{N}\left(0, \sigma^{2}\right)$. As análises de correlações entre as variáveis paramétricas foram estimadas pelo método de Pearson (Sampaio, 2002). As diferenças entre as médias dos tratamentos propostos foram comparadas em nível de 5\% de significância. Os resultados foram obtidos utilizando-se $\mathrm{o}$ programa SAS (User's..., 1999).

\section{RESULTADOS E DISCUSSÃO}

As médias das temperaturas de bulbo seco (BS) e bulbo úmido (BU) e a temperatura do globo negro (GN), bem como o cálculo do ITU dentro da câmara bioclimática foram avaliados para a caracterização do ambiente térmico durante o período experimental e os valores são apresentados na Tab. 1. As médias das temperaturas e do ITU permaneceram constantes dentro da câmara bioclimática, tanto no verão quanto no inverno, demonstrando linearidade desses valores e o funcionamento adequado dos aparelhos durante o período experimental. As temperaturas médias dos termômetros de bulbo seco, bulbo úmido e globo negro no período da manhã, tanto no verão quanto no inverno, foram, respectivamente, $22,18,23^{\circ} \mathrm{C}$, com ITU igual a 69, mostrando que os animais permaneceram dentro da faixa de conforto térmico. No período da tarde, esses valores médios apresentaram elevação significativa, $43, \quad 36$ e $44^{\circ} \mathrm{C}$, respectivamente, para os termômetros de bulbo seco, bulbo úmido e globo negro, e ITU igual a 97, colocando esses animais sob condições de 
estresse calórico severo. A temperatura do ar no período da tarde estava muito acima da temperatura crítica mais alta para bovinos, índice que representa, de modo geral, condição de emergência para os animais (Silva, 2000). Segundo Hahn (1985), citado por Silva (2000), o cálculo do ITU é válido para animais domésticos em geral e pode ser utilizado na avaliação do conforto térmico. Valores de ITU inferiores a 70 indicam condição não estressante, enquanto valores superiores a 83 indicam estresse severo.

Tabela 1. Temperaturas médias dos termômetros de bulbo seco (BS), bulbo úmido (BU) e globo negro $(\mathrm{GN})$, em diferentes pontos da câmara bioclimática, distribuídos na porta $(\mathrm{P})$, próximo à torneira $(\mathrm{T})$ e no cocho (C)

\begin{tabular}{ccccc}
\hline & \multicolumn{2}{c}{ Manhã $\left({ }^{\circ} \mathrm{C}\right)$} & \multicolumn{2}{c}{ Tarde $\left({ }^{\circ} \mathrm{C}\right)$} \\
\cline { 2 - 5 } & Inverno & Verão & Inverno & Verão \\
\hline BS-P & 22 & 22 & 43 & 43 \\
BS-T & 22 & 22 & 42 & 42 \\
BS-C & 22 & 22 & 44 & 44 \\
\hline Média & 22 & 22 & 43 & 43 \\
\hline BU-P & 18 & 18 & 36 & 36 \\
BU-T & 17 & 17 & 35 & 36 \\
BU-C & 18 & 18 & 36 & 36 \\
\hline Média & 18 & 18 & 36 & 36 \\
\hline GN-P & 23 & 23 & 43 & 44 \\
GN-T & 22 & 22 & 44 & 44 \\
GN-C & 23 & 23 & 44 & 44 \\
\hline Média & 23 & 23 & 44 & 44 \\
\hline ITU-P & 70 & 70 & 97 & 97 \\
ITU-T & 69 & 69 & 96 & 97 \\
ITU-C & 70 & 70 & 98 & 98 \\
\hline Média & 69 & 69 & 97 & 97
\end{tabular}

Para dar suporte à resposta fisiológica dos animais dentro da câmara bioclimática, na Tab. 2 encontram-se as médias dos valores de temperatura ambiente e umidade relativa do ar máxima, mínima e média nos meses de fevereiro, março e dezembro (verão) e junho e julho (inverno) do ano de 2004.
$\mathrm{Na}$ Tab. 3 verificam-se os valores médios da temperatura retal (TR), freqüência respiratória (FR) e temperatura da superfície corporal (TSC) nos períodos antes (manhã) e após o estresse (tarde) calórico, nas estações de verão e inverno. 
Tabela 2. Médias das temperaturas $\left({ }^{\circ} \mathrm{C}\right)$ ambientes (TA) e da umidade (\%) relativa (UR) segundo os meses, ano de 2004

\begin{tabular}{ccccccccccc} 
& \multicolumn{2}{c}{ Fevereiro } & \multicolumn{2}{c}{ Março } & \multicolumn{2}{c}{ Junho } & \multicolumn{2}{c}{ Julho } & \multicolumn{2}{c}{ Dezembro } \\
\hline & TA & UR & TA & UR & TA & UR & TA & UR & TA & UR \\
\hline Máx. & 29,0 & 77,1 & 29,2 & 69,7 & 25,0 & 83,1 & 24,0 & 82,9 & 29,5 & 77,9 \\
Min. & 17,5 & 67,3 & 16,9 & 60,0 & 12,5 & 59,4 & 12,8 & 61,9 & 16,6 & 66,9 \\
\hline Média & 23,3 & 72,2 & 23,1 & 64,9 & 18,7 & 71,3 & 18,4 & 72,4 & 23,1 & 72,4 \\
\hline
\end{tabular}

Tabela 3. Médias da temperatura retal $\left({ }^{\circ} \mathrm{C}\right)$, freqüência respiratória (mov/min) e temperatura da superfície corporal $\left({ }^{\circ} \mathrm{C}\right)$ antes (manhã) e após (tarde) o estresse calórico, no verão (VER) e no inverno (INV), de bovinos $1 / 2$ Gir x $1 / 2$ Holandês

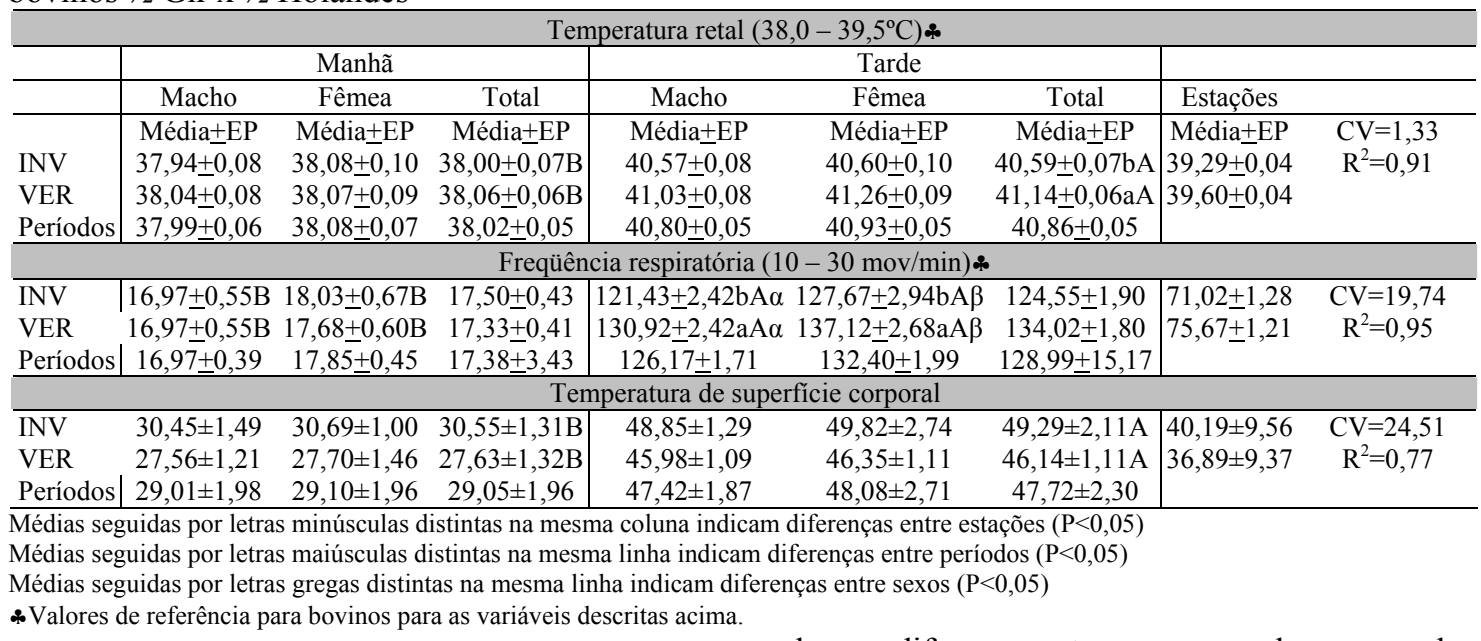

A TR no período da manhã apresentou-se dentro da faixa de normalidade para bovinos tanto no verão quanto no inverno $(\mathrm{P}>0,05)$ sendo a média entre as estações de $38,02^{\circ} \mathrm{C}$. Os valores na Tab. 3 mostram que, no período da tarde, a TR foi maior do que no período da manhã $(\mathrm{P}<0,05)$. No período da tarde, os valores de TR foram superiores aos limites fisiológicos, evidenciando que os animais não conseguiram manter a homeotermia. $\mathrm{O}$ armazenamento de calor pelo aumento da temperatura corporal da manhã para tarde resultou em hipertermia nos bovinos estressados, tanto na estação do inverno $\left(40,59^{\circ} \mathrm{C}\right)$ quanto no verão $\left(41,14^{\circ} \mathrm{C}\right)$.

Os valores médios da FR mostram que pela manhã esses se apresentaram dentro dos limites fisiológicos (17,50 e 17,33 $\mathrm{mov} / \mathrm{min})$, tanto na estação de inverno quanto na de verão. À tarde, essa freqüência apresentou aumento de aproximadamente sete vezes ultrapassando os limites fisiológicos. A FR dos animais no verão $(134,02 \mathrm{mov} / \mathrm{min})$ foi maior do que no inverno (124,55 mov/min). Apenas no período da tarde houve diferença entre sexos, sendo que machos apresentaram FR menor do que fêmeas, tanto na estação do inverno (121,43 e $127,67 \mathrm{mov} / \mathrm{min})$ quanto no verão $(130,92$ e $137,12 \mathrm{mov} / \mathrm{min})$ $(\mathrm{P}<0,05)$.

O aumento da TR e da FR acompanhou o aumento da temperatura ambiente dentro da câmara bioclimática. Para se defender do estresse térmico, os bovinos recorrem a mecanismos adaptativos fisiológicos de perda de calor corporal para evitar a hipertermia. Dessa forma, aumentam a freqüência respiratória (taquipnéia), como mecanismo adicional a perda de calor por sudorese, constituindo-se, ambos, em meios importantes de perda de calor por evaporação (Baccari Jr., 2001). Esses dados demonstram que, mesmo com esse grande aumento da freqüência respiratória, esses bovinos não conseguiram evitar a hipertermia.

A TSC aumentou significativamente no período da tarde $(\mathrm{P}<0,05)$, e não se observaram diferenças entre estações (Tab. 3). A média por períodos foi de $29,05{ }^{\circ} \mathrm{C}$ antes do estresse 
calórico e $47,72^{\circ} \mathrm{C}$ após o estresse calórico. O aumento da TSC reflete, diretamente, o aumento da temperatura ambiente, não caracterizando, portanto, a temperatura corporal dos animais. A TSC no período da tarde foi superior às relatadas pela literatura (Hansen, 1990; Maia, 2002; Morais, 2002; Azevedo, 2004). A diferença entre os resultados dos autores citados e os do presente estudo, deve-se, possivelmente, às temperaturas ambientes mais elevadas dentro da câmara bioclimática.

A diferença da TR do período da manhã para a tarde, foi de 2,6 e $3,1^{\circ} \mathrm{C}$ nas estações do inverno e verão, mostrando que essa diferença foi mais acentuada na estação do verão. A diferença da FR do período da manhã para tarde também se apresentou maior durante a estação do verão $(116,7 \mathrm{mov} / \mathrm{min})$ do que no inverno (106,1 mov/min). Temperaturas ambientais mais altas no verão (Tab. 2), associadas à elevação da temperatura corporal decorrente do aumento da temperatura dentro da câmara bioclimática favorecidas pelo estresse calórico agudo, foram os fatores responsáveis por este efeito.

A diferença entre as estações pode ser atribuída à adaptação dos animais às temperaturas ambientes. Como no verão, possivelmente, os animais estão apresentando maior oscilação destes parâmetros fisiológicos, a resposta, dentro da câmara bioclimática, será semelhante àquela que o animal vem apresentando no ambiente. Sabe-se que, no verão, a TR e a FR são significativamente superiores às do inverno. No verão há aumento da temperatura do ar, da umidade e da radiação solar promovendo sobrecarga térmica radiante dificultando a dissipação de calor o que resulta no aumento da temperatura corporal dos animais. Como resultado, a TR é substancialmente elevada (Nardone, 1998; Pires, 1997).

A TR encontra-se positivamente correlacionada com a FR $(\mathrm{r}=0,92 ; \quad \mathrm{P}<0,001)$. Correlações positivas e significativas entre TR e FR em bovinos também foram observadas por Dunlap e Vicent (1971) e por Azevedo (2004). Este achado é esperado uma vez que o bovino, em altas temperaturas ambientais, lança mão do sistema respiratório para perder calor, visando evitar o aumento da temperatura corporal. Não se observou correlação entre a TR ou entre a FR com a TSC. A TSC pode ser um dos parâmetros de mensuração para o aumento da temperatura corporal, mas deve-se salientar que este não reflete precisamente as alterações da temperatura corporal como são observados na mensuração da temperatura retal e da temperatura da membrana timpânica (Damanhouri e Tayeb, 1992). A análise de variância revelou interação entre época e período para a TR e a FR. Essa interação reflete o maior aumento dessas variáveis na estação do verão que no inverno.

\section{CONCLUSÕES}

Conclui-se que os animais apresentam aumento nas variáveis fisiológicas indicadoras de estresse calórico severo quando mantidos por seis horas em câmara bioclimática a $42^{\circ} \mathrm{C}$ e $60 \%$ de umidade e que a temperatura retal e a freqüência respiratória são importantes parâmetros para avaliar o estresse calórico em bovinos.

\section{REFERÊNCIAS BIBLIOGRÁFICAS}

AZEVEDO, M. Efeitos do verão e do inverno sobre os parâmetros fisiológicos de vacas mestiças Holandês-Zebu, em lactação, na região de Coronel Pacheco, MG. 2004. 85f. Tese (Doutorado) - Escola de Veterinária, Universidade Federal de Minas Gerais, Belo Horizonte.

BACCARI Jr., F. A temperatura corporal dos bovinos. Gado Holandês, n.51, p.15-19, 1987.

BACCARI Jr., F. Estudo da freqüência cardíaca e da temperatura retal em bovinos leiteiros da espécie Bos taurus. I. Efeito da idade. II. Grupos etários. Valores normais em condições naturais de clima tropical. III. Comparação entre raças, sexos e períodos do dia. Arq. Esc. Vet. UFMG, v.23, p.337, 1971.

BACCARI Jr., F. Manejo ambiental da vaca leiteira em climas quentes. Londrina: Editora da Universidade Estadual de Londrina, 2001. 142p.

BIANCA, W. Reviews of the progress of dairy science. Section A. Physiology. Cattle in a hot environmental. $J$. Dairy Res., v.32, p.291-345, 1965.

CARVALHO, F.A.; LAMMOGLIA, M.A.; SIMÕES, M.J. et al. Breed effects thermoregulation and epithelial morphology in imported and native cattle subjected to heat stress. J. Anim. Sci., v.73, p.3570-3573, 1995.

CUNNINGHAM, J.G. Tratado de fisiologia veterinária. 2.ed. Rio de Janeiro: Guanabara Koogan, 1999. 454p.

DIRKSEN, G.; GRÜNDER, H.D.; STÖBER, M. Exame clínico dos bovinos 3.ed. Rio de Janeiro: Guanabara Koogan, 1993. 419p. 


\section{Ferreira et al.}

DUNLAP, S.E.; VICENT, C.K. Influence of postbreeding thermal stress on conception rate in beef cattle. J. Anim. Sci., v.32, p.1216-1218, 1971 .

DuPREEZ, J.H. Parameters for the determination and evaluation of heat stress in dairy cattle in South Africa. Onderstepoort J. Vet. Res., v.67, p.263-271, 2000.

IGONO, M.G.; STEEVENS, B.J.; SHANKLIN, M.D. et al Spray cooling effects on milk production, milk and rectal temperatures of cows during a moderate temperature summer season. J. Dairy Sci., v.68, p.979-985, 1985.

INGRAHAM, R.H.; STANLEY, R.W.; WAGNER, W.C. Seasonal effects on shade and nonshade cows as measure by rectal temperature, adrenal cortex hormones, thyroid hormone and milk production. Am. J. Vet. Res., v.40, p.1792$1797,1979$.

KELLY, C.F.; BOND, T.E. Bioclimatic factors and their measurements. In: NATIONAL ACADEMIC OF SCIENCES (Ed.). A guide to environmental research on animals. Washington: National Academic of Sciences, 1971. p.71-92.

MAIA, A.S.C. Variação genética e ambiental das características morfológicas e das propriedades radiativas efetivas do pelame de bovinos da raça holandesa. 2002. $77 \mathrm{f}$ Dissertação (Mestrado) - Faculdade de Ciências Agrárias e Veterinárias, Universidade Estadual Paulista, Jaboticabal, SP.

MARAI, I.F.M.; HABEEB, A.A.M.; FARGHALY, H.M. Productive, physiological and biochemical changes in imported an locally born Holstein lactating cows under hot summer conditions of Egypt. Trop. Anim. Health Prod., v.31, p.233-243, 1999.

MARTELLO, L. S. Diferentes recursos de climatização e sua influência na produção de leite, na termorregulação dos animais e no investimento das instalações. 2002. 67f. Dissertação (Mestrado) - Faculdade de Zootecnia e Engenharia de Alimentos, Universidade de São Paulo, Pirassununga, SP.

McDOWELL, R.E. Determining the suitable of livestock to warm climates. In: . Improvement of livestock production in warm climates. San Francisco: W.H. Freeman, 1972. 432p.

MORAIS, D.A.E.F. Comportamento anual de característica de pelame, níveis de hormônios tireoideanos e produção de leite de vacas mestiças em ambiente quente e seco. 2002. 123f. Tese (Doutorado) - Faculdade de Ciências Agrárias e Veterinária, Universidade Estadual Paulista, Jaboticabal, SP.

MOTA, L. S. Adaptação e interação genótipo-ambiente em vacas leiteiras. 1997. 69f. Tese (Doutorado) - Faculdade de Medicina de Ribeirão Preto, Universidade de São Paulo, Ribeirão Preto, SP

MULLER, C.J.; BOTHA, J.A. Effect of summer climatic conditions on different heat tolerance indicators in primiparous Friesian and Jersey cows. S. Afr. J. Anim. Sci., v.23, p.98-103, 1993

MULLER, C.J.; BOTHA, J.A.; COETZER, W.A. et al. Effect of shade on various parameters of Friesian cow in a Mediterranean climate in South Africa. 2: Physiological responses. S. Afr. J. Anim. Sci., v.24, p.56-60, 1994a.
MULLER, C.J.C.; BOTHA, J.A.; SMITH, W.A. et al. Production, physiological and behavioral responses of lactating Friesian cows to a shade structure in a temperate climate. IN: BUCKLIN, R.A. (Ed.). INTERNATIONAL DAIRY HOUSING CONFERENCE, 3.,1994b, St. Joseph, MI. Proceeding... St. Joseph, MI: American Society of Agricultural Engineers, 1994b. p.597-588

NARDONE, A. Thermoregulatory capacity among selection objectives in dairy cattle in hot environment. Zootec. Nutr. Anim., v.24, p.295-306, 1998.

OKANTAH, S.A.; AGGREY, S.E.; AMOAKO, K.J. The effect of diurnal changes in ambient temperature on heat tolerance in some cattle breeds and crossbreeds in a tropical environment. Bull. Anim. Health Prod. Afr., v.41, p.33-38, 1992

PIRES, M.F.A. Comportamento, parâmetros fisiológicos e reprodutivos de fêmeas da raça Holandesa confinadas em free-stall, durante o verão e o inverno. 1997. 151f. Tese (Doutorado) - Escola de Veterinária, Universidade Federal de Minas Gerais, Belo Horizonte.

PIRES, M.F.A.; FERREIRA, A.M.; COELHO, S.G. Estresse calórico em bovinos de leite. In: SIMPÓSIO DE PRODUÇÃO E NUTRIÇÃO DE GADO LEITEIRO, 1998a, Belo Horizonte. Anais... Belo Horizonte, 1998a. p.17-30

PIRES, M.F.A.; SATURNINO, H.M.; VERNEQUE, R.S. et al. Efeito das estações (inverno e verão) na temperatura retal e freqüência respiratória de vacas Holandesas confinadas em free stall. Arq. Bras. Med. Vet. Zootec., v.50, p.747-752, $1998 b$.

ROBINSON, E.N. Termorregulação. In: CUNNINGHAM, J.G. Tratado de fisiologia veterinária. 2.ed. Rio de Janeiro: Guanabara Koogan, 1999. cap.51, p.427-435.

SAMPAIO, I.B.M. Estatística aplicada à experimentação animal. 2.ed. Belo Horizonte: Fundação de Ensino e Pesquisa em Medicina Veterinária e Zootecnia, 2002. 265p.

SILVA, R.G. Introdução à bioclimatologia animal. São Paulo: Nobel, 2000. 286p

STÖBER, M. Identificação, anamnese, regras básicas da técnica de exame clínico geral. In: DIRKSEN, G.; GRÜNDER, H.D.; STÖBER, M. Exame clínico dos bovinos. 3.ed. Rio de Janeiro: Guanabara Koogan, 1993. cap.2, p.4480

TERRA, R.L. História, exame físico e registro dos ruminantes. In: SMITH, B.P. Tratado de medicina interna dos grandes animais. São Paulo: Manole, 1993. v.1, cap.1, p.3-15.

USER'S guide: statistics. Version 8. Cary, NC: SAS Institute, 1999

WOLFF, L.K.; MONTY, D.E. Physiologic responses to intense summer heat stress and its effect on the estrous cycle of non-lactating and lactating Holstein-Friesian cows in Arizona. Am. J. Vet. Res., v.35, p.187-192, 1974. 